\title{
Acute on Chronic Immune Thrombocytopenia as a Cause of Thrombocytopenia in Sepsis
}

\author{
Pujitha Kudaravalli ${ }^{1}$, Venkata Satish Pendela ${ }^{2}$, Harvir Singh S. Gambhir ${ }^{1}$ \\ 1. Internal Medicine, State University of New York (SUNY) Upstate Medical University, Syracuse, USA 2. Internal \\ Medicine, Rochester General Hospital, Rochester, USA
}

Corresponding author: Pujitha Kudaravalli, kudaravp@upstate.edu

\begin{abstract}
Immune thrombocytopenia (ITP) is a diagnosis of exclusion and can be challenging at times to make the diagnosis. We herein present a case of a 73-year-old male with a history of chronic ITP, who presented to the hospital with sepsis and developed thrombocytopenia. His thrombocytopenia did not improve with resolution of sepsis but improved with ITP treatment including immunoglobulins and steroids. Plateletassociated IgG antibody levels are inversely proportional to platelet counts. The antibody levels are increased in sepsis. We would like to highlight that other causes of thrombocytopenia should also be considered in sepsis.
\end{abstract}

Categories: Internal Medicine, Hematology

Keywords: immune thrombocytopenia, sepsis, immunoglobulins, steroids

\section{Introduction}

Immune thrombocytopenia (ITP) is a diagnosis of exclusion, with an incidence of two per 100,000 adults with a median age of 50 [1]. Most adults have chronic ITP and the pathogenesis of ITP continues to remain unclear. We herein present a case of acute on chronic ITP in a patient with sepsis who responded to ITP treatment with steroids and intravenous immunoglobulin. We would like to highlight that acute on chronic ITP should be considered as a cause of thrombocytopenia in sepsis in patients with chronic ITP as there is also increased production of platelet-associated immunoglobulin $\mathrm{G}$ antibodies during sepsis.

\section{Case Presentation}

A 73-year-old male presented to the ED with fever and lethargy for one day. His past medical history is significant for hyperlipidemia, hypertension, gout, and chronic ITP. His past surgical history included a recent dental procedure with a dental implant placement.

Received 05/01/2020 Review began 05/07/2020 Review ended 05/08/2020 Published 05/17/2020

() Copyright 2020

Kudaravalli et al. This is an open access article distributed under the terms of the Creative Commons Attribution License CC-BY 4.0., which permits unrestricted use, distribution, and reproduction in any medium, provided the original author and source are credited.
On examination, his temperature was 39.3 degree Celsius, blood pressure was 137/89 mmHg, heart rate was 99 beats/min. Cardiovascular system and respiratory system were unremarkable. Laboratory findings showed a white blood cell (WBC) count of $3600 / \mathrm{mm}^{3}$, hemoglobin of $12.2 \mathrm{~g} / \mathrm{dL}$ with a hematocrit of $36.3 \%$, and platelet count of $45000 / \mathrm{mm}^{3}$. The baseline platelet count of the patient was $90000 / \mathrm{mm}^{3}$. Chest X-ray (as shown in Figure 1), respiratory panel, and urinalysis were negative for infection. Blood cultures did not show any growth. CT maxillofacial without contrast showed metallic implant in the left maxillary tooth and moderate to severe paranasal sinusitis (as shown in Figure 2). The patient was diagnosed with sepsis secondary to transient bacteremia secondary to dental procedure versus sinusitis and was started on broadspectrum antibiotics. 


\section{Cureus}

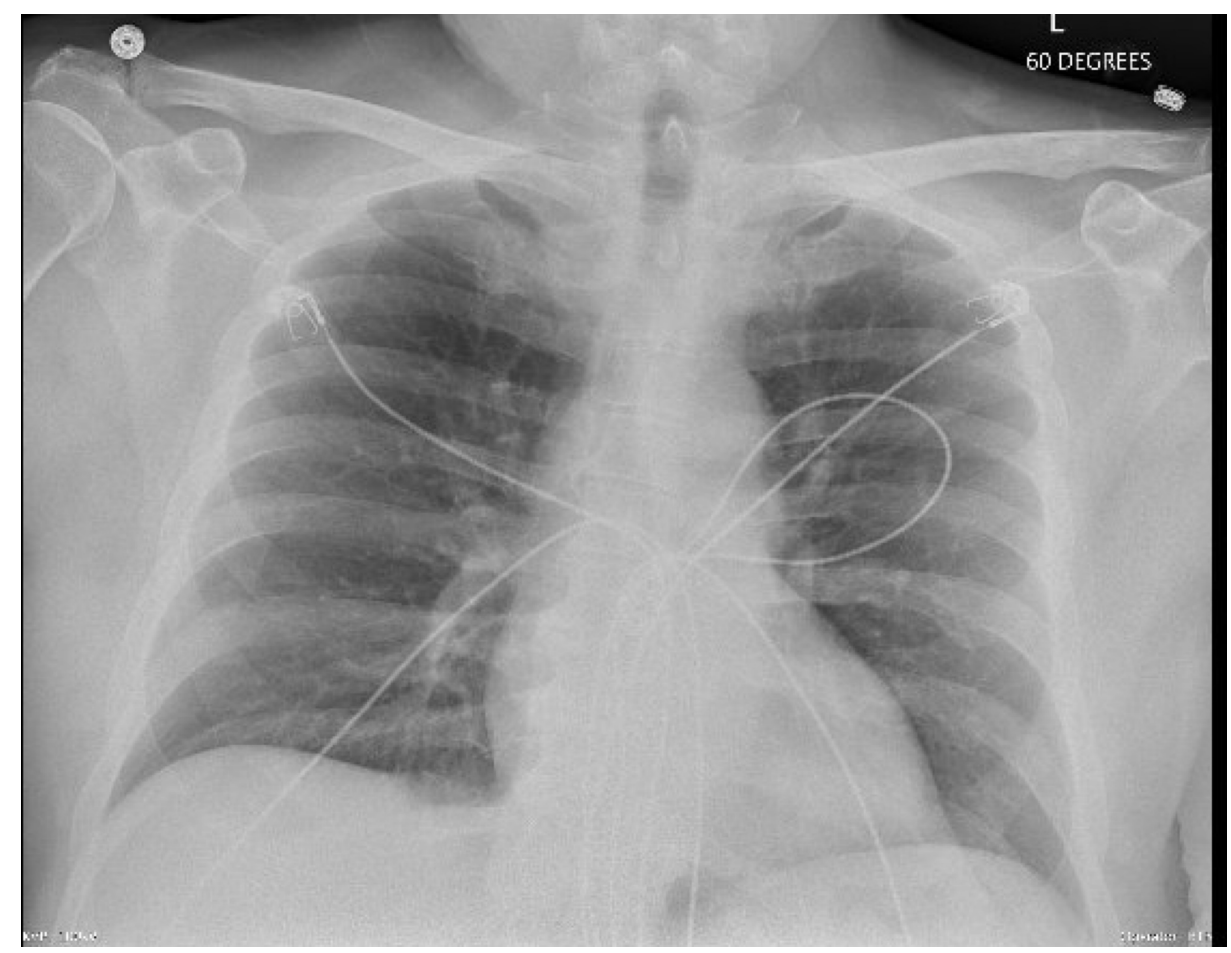

FIGURE 1: Chest X-ray.

Posterior anterior view of the X-ray of the chest showing no features of acute infection

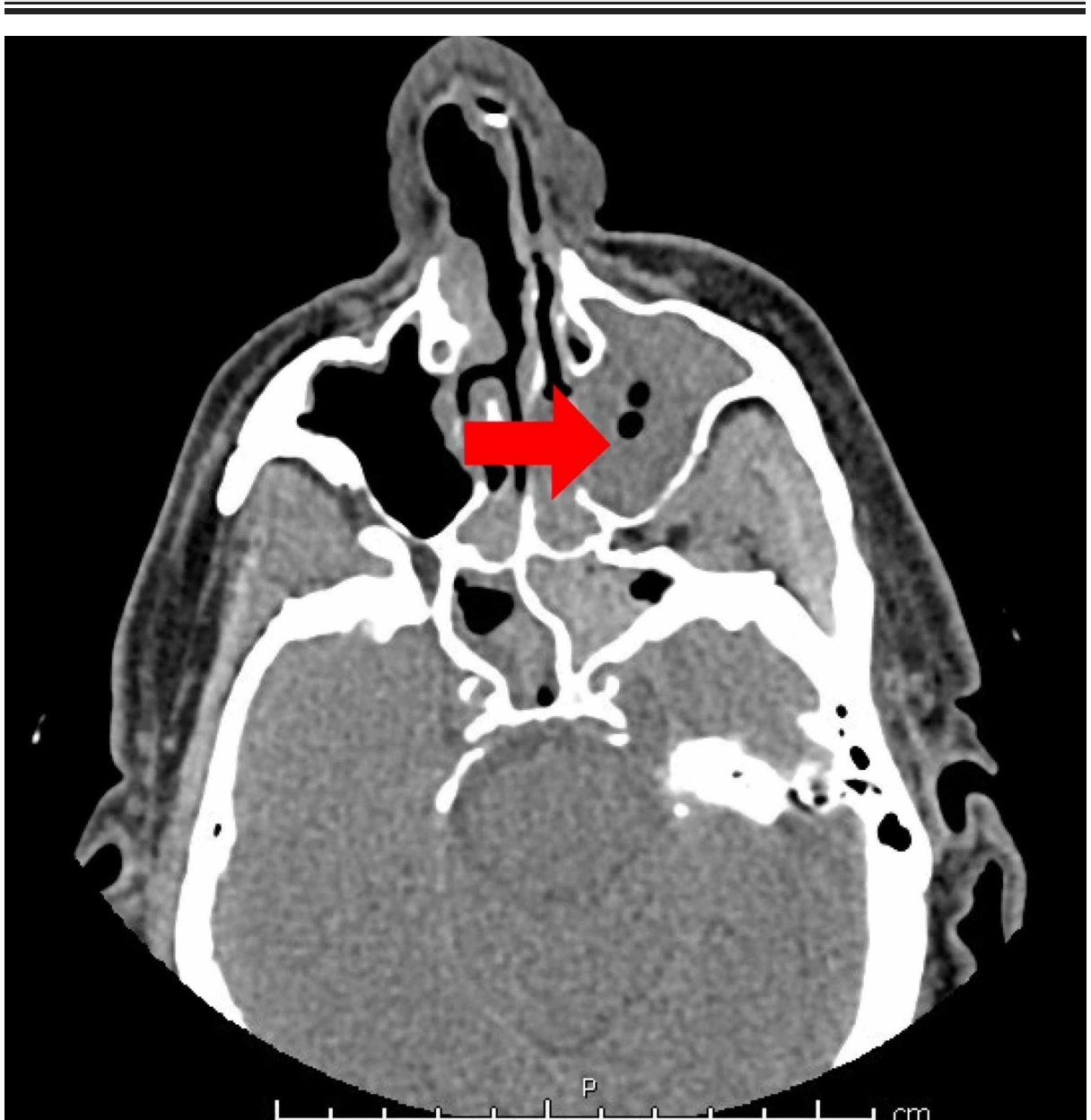

FIGURE 2: CT maxillofacial without contrast. 
The patient improved clinically with antibiotics and the signs of active infection resolved. However, his platelet count continued to worsen with a nadir of $25000 / \mathrm{mm}^{3}$. Hematology was consulted and the peripheral blood smear was reviewed which was unremarkable with no hemolysis. Other causes of thrombocytopenia such as infection with HIV, hepatitis C, autoimmune panel, thyroid stimulating hormone (TSH) were ruled out. Of note, the patient had a bone marrow biopsy nine months before presentation which showed 5.6\% plasma cells without evidence of clonality and high-normal number of megakaryocytes with thrombocytopenia consistent with peripheral destruction or sequestration confirming his previous diagnosis of chronic ITP. A presumptive diagnosis of acute on chronic ITP was made. He was started on IV immunoglobulins $1 \mathrm{mg} / \mathrm{kg}$ of ideal body weight before considering an immunosuppressant in a patient with infection and there was an improvement in platelet counts from $25000 / \mathrm{mm}^{3}$ to $41000 / \mathrm{mm}^{3}$. He was subsequently started on prednisone $100 \mathrm{mg}$ and his platelets drastically improved to $120000 / \mathrm{mm}^{3}$. He was discharged on prednisone $100 \mathrm{mg}$ PO daily for 7 days with an outpatient hematology follow up.

\section{Discussion}

Platelets are an integral part of the immune response, inflammation, pathogen killing, and tissue repair in sepsis in addition to hemostasis and thrombosis. Thrombocytopenia is commonly seen in sepsis and is also an important marker of prognosis in sick ICU patients. In a study done by Venkata et al., it was found that $47.6 \%$ of patients with sepsis had thrombocytopenia. Development of thrombocytopenia in sepsis is attributed to consumption from sequestration and destruction, immune-mediated mechanisms secondary to nonspecific platelet-associated antibodies, and cytokine-driven hemophagocytosis of platelets [23]. Thrombocytopenia in sepsis is also caused by decreased platelet production in the bone marrow caused by inhibitory effects of toxins, drugs, or inflammatory mediators.

Persistent thrombocytopenia is associated with increased mortality and longer ICU stay [2, 4]. It is also observed that patients with thrombocytopenia are more sick [5]. The most common practice of management for thrombocytopenia in sepsis is treatment of underlying infection and continued monitoring but it is important to recognize that in a patient with chronic ITP, thrombocytopenia can be secondary to an exacerbation of underlying ITP. Immune system may be involved in the reduction in platelet count in septicemia. In a study done by Matschke et al., it was shown that platelet associated IgG antibodies were elevated in septic patients and their levels were inversely proportional to the platelet counts [6].

Immune thrombocytopenia can be primary or secondary, acute (less than six months) or chronic. Secondary forms of the disease are thrombotic thrombocytopenic purpura, hemolytic uremic syndrome, disseminated intravascular coagulation, paroxysmal nocturnal hemoglobinuria, infection with hepatitis $\mathrm{C}$ and HIV, drugs like heparin and quinidine, lymphoproliferative disorders like chronic lymphocytic leukemia. A patient is diagnosed with ITP after the above differentials are ruled out. It is hypothesized that there is development of immunoglobulin $\mathrm{G}$ antibodies targeting platelet membrane glycoprotein IIb-IIIa. These platelets that are coated with IgG antibodies undergo accelerated destruction in the liver/spleen or intramedullary destruction of antibody coated platelet by macrophages $[4,7]$.

The first line of treatment for ITP is prednisone $1 \mathrm{mg} / \mathrm{kg}$ for two to four weeks. Repeated cycles of pulsed high dose dexamethasone have also shown similar results as that of daily prednisone therapy [8]. Splenectomy was a popular treatment for ITP but recent data show that $<20 \%$ of patients now undergo splenectomy for ITP. Anti-D immunoglobulin therapy has also demonstrated efficacy in rapidly increasing platelet counts in patients with an intact spleen and it used to delay splenectomy in adults. It has an advantage over intravenous immunoglobulin (IVIG) with regard to expense and longer duration of action [9]. Rituximab, thrombopoietin receptor agonist (TPO-RA), and IVIG are other treatment modalities. Several novel therapies are on the horizon for ITP including antibodies targeting CD40-CD154 interaction between $\mathrm{B}$ and $\mathrm{T}$ cells, novel agents increasing platelet production, including amifostine, treatments targeting the Fc receptor [10].

\section{Conclusions}

We would like to highlight that acute on chronic ITP should be considered as a cause of thrombocytopenia in patients with chronic ITP when they are admitted to the hospital with sepsis. Not all thrombocytopenia in patients can be related to sepsis and other causes should also be considered. The treatment of ITP is different from that of sepsis-induced thrombocytopenia and physicians should be vigilant of this condition.

\section{Additional Information}

\section{Disclosures}

Human subjects: Consent was obtained by all participants in this study. N/a issued approval n/a. n/a. 
Conflicts of interest: In compliance with the ICMJE uniform disclosure form, all authors declare the following: Payment/services info: All authors have declared that no financial support was received from any organization for the submitted work. Financial relationships: All authors have declared that they have no financial relationships at present or within the previous three years with any organizations that might have an interest in the submitted work. Other relationships: All authors have declared that there are no other relationships or activities that could appear to have influenced the submitted work.

\section{References}

1. Zainal A, Salama A, Alweis R: Immune thrombocytopenic purpura. J Commun Hosp Intern Med Perspect. 2019, 9:59-61.

2. Venkata C, Kashyap R, Farmer JC: Thrombocytopenia in adult patients with sepsis: incidence, risk factors, and its association with clinical outcome. J Intensive Care. 2013, 1:9. 10.1186/2052-0492-1-9

3. Ghosh TK, Khan N, Malik A: Platelet autoantibodies in septicaemic patients. Indian J Pathol Microbiol. 1999, 42:31-35.

4. Vanderschueren S, De Weerdt A, Malbrain M: Thrombocytopenia and prognosis in intensive care. Crit Care Med. 2000, 28:1871-1876. 10.1097/00003246-200006000-00031

5. Kelton JG, Neame PB, Gauldie J : Elevated platelet-associated IgG in the thrombocytopenia of septicemia . N Engl J Med. 1979, 300:760-764. 10.1056/NEJM197904053001404

6. Matschke J, Muller-Beissenhirtz H, Novotny J: A randomized trial of daily prednisone versus pulsed dexamethasone in treatment-naïve adult patients with immune thrombocytopenia: EIS 2002 Study. Acta Haemotol. 2016, 136:101-107. 10.1159/000445420

7. Cines DB, Blanchette S: Immune thrombocytopenic purpura. N Engl J Med. 2002, 346:10.1056/NEJMra010501

8. Dewitte A, Lepreux S, Ripoche J: Blood platelets and sepsis pathophysiology: a new therapeutic prospect in critical ill patients?. Ann Intensive Care. 2018, 28:32. 10.1186/s13613-017-0337-7

9. Cheung E, Liebman HA: Anti-RhD immunoglobulin in the treatment of immune thrombocytopenia . Biologics. 2009, 3:57-62.

10. Lambert MP, Gernsheimer TB: Clinical updates in adult immune thrombocytopenia. Blood. 2017, 129:28292835. 10.1182/blood-2017-03-754119 